See discussions, stats, and author profiles for this publication at: https://www.researchgate.net/publication/313784044

\title{
Multivariate Functional Network Connectivity for Disorders of Consciousness
}

Conference Paper in Lecture Notes in Computer Science · February 2017

DOI: 10.1007/978-3-319-52277-7_-53

\section{CITATIONS}

0

9 authors, including:

Jorge Rudas

National University of Colombia

17 PUBLICATIONS 14 CITATIONS

SEE PROFILE

A. Athena Demertzi

University of Liège

147 PUBLICATIONS 4,231 CITATIONS

SEE PROFILE
READS

124

12. Darwin Martinez

16 PUBLICATIONS 2 CITATIONS

SEE PROFILE

6. Carol Di Perri

Coma Science Group, University of Liege, Liege, Belgium 72 PUBLICATIONS 966 CITATIONS

SEE PROFILE

Some of the authors of this publication are also working on these related projects:

hypnosis and pain View project

Characterization of interactions between resting state networks for disorders of consciousness View project 


\title{
Multivariate functional network connectivity for disorders of consciousness
}

\author{
Jorge Rudas ${ }^{1}$, Darwin Martínez ${ }^{2,3}$, Athena Demertzi ${ }^{4,5}$, \\ Carol Di Perri ${ }^{5}$, Lizette Heine ${ }^{5}$, Luaba Tshibanda ${ }^{5}$, Andrea Soddu ${ }^{6}$, \\ Steven Laureys ${ }^{5}$, and Francisco Gómez ${ }^{7}$ \\ 1 Department of Biotechnology, Universidad Nacional de Colombia \\ 2 Department of Computer Science, Universidad Nacional de Colombia \\ 3 Department of Computer Science, Universidad Central, Colombia \\ ${ }^{4}$ Institut du Cerveau et de la Moelle épinière, Hôpital de la Pitié-Salpêtrière, France \\ ${ }^{5}$ Coma Science Group, GIGA Research Center, University of Liège, Belgium \\ ${ }^{6}$ Department of Physics and Astronomy, Western University, Canada \\ 7 Department of Mathematics, Universidad Nacional de Colombia
}

\begin{abstract}
Recent evidence suggests that healthy brain is organized on large-scale spatially distant brain regions, which are temporally synchronized. These regions are known as resting state networks (RSNs). The level of interaction among these functional entities has been studied in the so called functional network connectivity (FNC). FNC aims to quantify the level of interaction between pairs of RSNs, which commonly emerge at similar spatial scale. Nevertheless, the human brain is a complex functional structure which is partitioned into functional regions that emerge at multiple spatial scales. In this work, we propose a novel multivariate FNC strategy to study interactions among communities of RSNs, these communities may emerge at different spatial scales. For this, first a community or hyperedge detection strategy was used to conform groups of RSNs with a similar behavior. Following, a distance correlation measurement was employed to quantify the level of interaction between these communities. The proposed strategy was evaluated in the characterization of patients with disorders of consciousness, a highly challenging problem in the clinical setting. The results suggest that the proposed strategy may improve the capacity of characterization of these brain altered conditions.
\end{abstract}

Keywords: Community, disorders of consciousness, hyperedge, multivariate functional network connectivity, resting state networks

\section{Introduction}

After severe brain injury, some patients may fall in coma. After coma, some of them may evolve to severely altered states of consciousness, such as, Vegetative State/Unresponsive Wakefulness Syndrome (VS/UWS), in which patients open their eyes but remain unresponsive to external stimuli [1], or Minimally 
Conscious State (MCS), in which patients exhibit signs of fluctuating yet reproducible remnants of non-reflex behavior [2]. These conditions are collectively known as Disorders of Consciousness (DOCs).

In recent years, a novel neuroimaging protocol called resting state fMRI (functional Magnetic Resonance Imaging) has been used to study brain activity in pathological/pharmacological altered brain conditions. In this protocol, the subject rests in a magnetic resonator few minutes without being exposed to any stimuli, while their hemodynamic brain activity is recorded. Using this protocol, a sets of spatial regions with common functional behaviors have been identified. The different set of regions have been called Resting State Network (RSN) [3]. At least ten of these entities have been consistently identified in healthy subjects (default mode network (DMN), executive control network left (ECL), executive control network right (ECR), saliency, sensorimotor, auditory, cerebellum and three visual networks medial, lateral and occipital). These networks have been linked to cognitive/sensorial high level processes [3]. Recently, the interactions among these RSNs have been studied by using the so called functional network connectivity (FNC). FNC have been used to study brain dynamic during altered brain conditions, including, pharmacological alterations, pathological conditions and brain reconfiguration in response to external task modulations, among others [4-6]. Particularly, for DOC conditions it has been suggested that a dysfunctional time-sustained hyper-connectivity between executive control left (ECL) - executive control right (ECR) and visual medial - salience networks may be linked to the severe states of loss of consciousness [7]. In addition, the level of modularity between RSNs has been shown to be reduced in patients with DOC in comparison with healthy subjects [8]. Nevertheless, despite of recent advances in the description of these brain dynamics for patients in DOC conditions, a complete characterization of these neurological alterations remains as a major clinical challenge [9].

FNC aims to assess the level of interactions between spatially remote neurophysiological events. These interactions are typically studied for brain regions of similar spatial scale [10]. However, the human brain is a complex functional structure that may be partitioned into regions that will emerge at multiple spatial scales [11]. For instance, brain activity may result from the interaction of large scale regions, such as, lobes or large functional systems (visual system, default mode network, etc) and small scale regions (Broca's area, hypothalamus and thalamus). These interactions across different spatial scales remains poorly studied [11]. The understanding of this phenomenon can potentially improve our knowledge about the DOC conditions [8].

Recently, the concept of community has been proposed to capture the segregation and integration phenomena, two major features that have been described for the brain activity in resting state [12]. Segregation refers to the organization of the brain dynamic in groups of specialized behavior, and integration is related to the existence of high levels of integration among some of these regions [12]. These two concepts have been used to group brain regions resulting in the concept of brain communities[12]. On the other hand, an extension of traditional 
graph approach also has been used in order to study the groups emerging of fMRI data, particularly, it has been extended the edge formulation to hyperedge, which the relation among the nodes into graph may be associated to more two nodes $[13,14]$. These hyperedges then, are similar to community definition, because, are groups of brain entities based an particular association measure. Recent evidence suggests that segregation and integration properties may be altered in DOC condition [8]. In this work, we propose a novel strategy to quantify the functional interaction between RSN brain communities or hyperedge. The proposed strategy accounts for linear and non-linear interactions between these entities, and considers the possibility of having communities/hyperedge emerging at different spatial scales. The proposed strategy was evaluated in the characterization of patients with DOC.

\section{Materials and Methods}

Figure 1 illustrates the proposed method. First, the fMRI data was preprocessed to account for the anatomical variability of the subjects (Section 2.2). Following, the individual fMRI resting state signal was decomposed into functional spatio-temporal components by using Independent Component Analysis (ICA). Later, an automatic matching procedure was applied to identify the set of functional components that corresponded to a set of predefined RSNs templates (Section 2.2). Then, a multi-objective optimization strategy was used to find a set of communities/hyperedge among these RSNs (Section 2.3). Finally, the interactions between these groups were quantified by using a distance correlation measurement (Section 2.4).

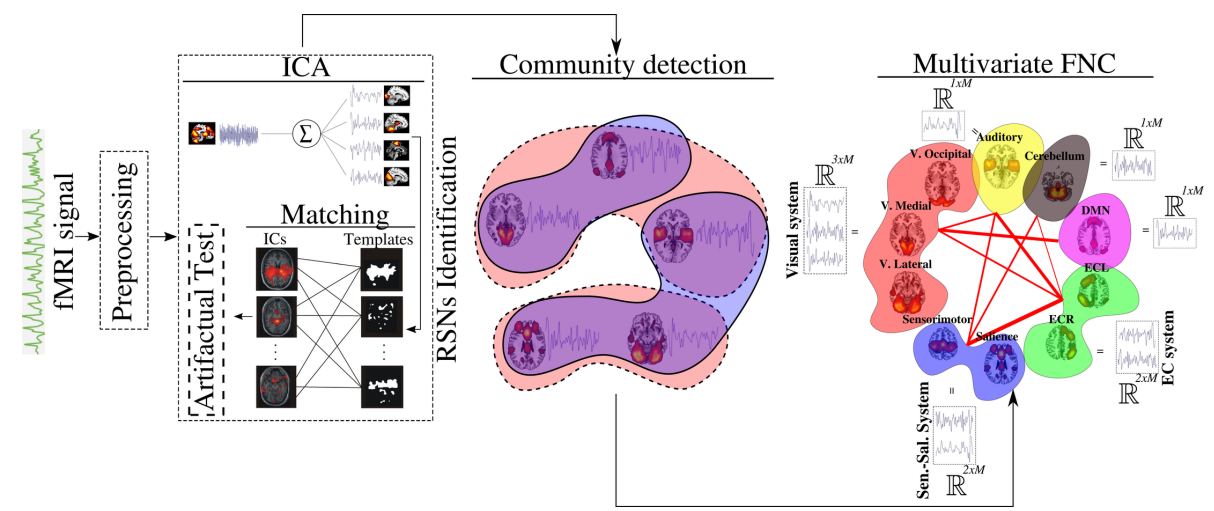

Fig. 1. Multivariate FNC approach. Firstly, the fMRI data were preprocessed to remove anatomical variability. Following, the fMRI signal was decomposed into functional components by using Independent Component Analysis (ICA). Later, a RSN identification procedure was applied on the resulting components to identify a set of RSNs. Subsequently, a multi-objective optimization method was used to find a set of RSN communities/hyperedge. Finally, the interaction among these groups was computed by using distance correlation. 


\subsection{Participants and data acquisitions}

Data from 76 subjects were used for this study: 27 healthy controls (14 women, mean age $47 \pm 16$ years), 24 patients in minimally conscious state and 25 with vegetative state/unresponsive wakefulness syndrome (20 women, mean age $50 \pm$ 18 years). All patients were clinically examined using the French version of the Coma Recovery Scale Revised (CRS-R) [15]. For each subject, fMRI resting data were acquired in a 3T scanner (Siemens medical Solution in Erlangen, Germany). Three hundred fMRI volumes multislice $T 2^{*}$-weighted functional images were captured (32 slices; voxel size: $3 \times 3 \times 3 \mathrm{~mm}^{3}$; matrix size 64 ; repetition time $=2000 \mathrm{~ms}$; echo time $=30 \mathrm{~ms}$; flip angle $=78$; field of view $=192 \mathrm{~mm}^{2}$ ). A structural T1 image was also acquired for anatomical reference. More details of the subjects demography can be found in [16]. These data are used exclusively for research of our collaborators.

\section{2 fMRI preprocessing}

fMRI data was processed using SPM8 ${ }^{8}$. Preprocessing included: realignment, coregistration of functional onto structural data, segmentation of structural data, normalization into MNI space and spatial smoothing with a Gaussian kernel of $8 \mathrm{~mm}$. Large head motions were corrected using ArtRepair ${ }^{9}$.

RSNs Identification The first step for the RSN identification was the fMRI signal decomposition into sources of neuronal/physiological origin. For this task, we used ICA, which aims to decompose the signal into a set of statistically independent components (ICs) of brain activity. In traditionally ICA, one considers the mixture as linear and the sources as statistically mutually independent and non-Gaussian [17]. In the fMRI data, the spatial dimension is much greater than temporal one, then, we used spatial ICA (sICA), which decompose the signal into maximally independent spatial maps [18]. In sICA each spatial map (source) have an associated time course, which corresponds to the common dynamic exhibit by this component. The RSNs time-courses obtained with sICA were subsequently used for all the FNC computations. For the sICA decomposition 30 components were used, this selection was performed based on previous work that have shown that this number of components is enough to characterize the different RSNs both for healthy controls and patients with DOC [16]. After the sICA decomposition, the different RSNs were identified at individual level. A machine learning based labeling method was applied to discriminate between IC of "neuronal" or "artifactual" origin [16].

\subsection{Community detection}

A community in graph theory refers to a groups of nodes which share common properties within a graph [12]. This definition naturally assumes the existence of

\footnotetext{
${ }^{8}$ http:www.fil.ion.ucl.ac.uk/spm

${ }^{9}$ http://cibsr.stanford.edu/tools/ArtRepair/ArtRepair.htm
} 
an underlying graph representation, i.e., the existence of a binary relation which characterizes the interaction between pairs of RSNs. This assumption may oversimplify the brain dynamic [8]. In order to account for this limitation, the notion of community can be reformulated to be not dependent of the graph representation. For our case, a community was defined as the set of nodes that share some commons feature or properties, similarly to hyperedge definition into hyper graph theory [8]. By using this formulation, the RSN community detection problem can be reformulated as a clustering problem. For this case, a segregationmodularity measurement was used to quantify the level of integration for groups of RSNs. This measure guided the clustering process. More particularly, two measures were used to quantify the level of segregation of a specific partition of the RSNs set [8]. The first measure, aims to quantify the level of information of each community by using a similar idea to the Kapur criterion, i.e., maximize the sum of entropy for all possible communities, high values for this measure can be expected when each community contains highly informative RNSs [19]. The second measure, considers the combination between both inter-community and intra-community variances by using the Otsu criterion [20]. These two measures were used in a multi-objective optimization approach to find the optimal partition that maximizes the brain segregation for the set of RSNs [8]. This process was applied to the data and a community model was obtained for the DOC subjects [8]. This model was composed by six communities: auditory, cerebellum, DMN, executive control system (ECN left and right), sensorimotor-salience and visual system (visual occipital, medial and lateral).

\subsection{Multivariate FNC method}

After the RSN community construction, each one is composed by one or more RSNs. Therefore, each community can be described by one or more time-courses. In order to quantify the level of interaction between different communities a distance correlation (DC) measure was used [21]. DC aims to quantify the level of non-linear interaction between two time series of arbitrary dimension [21]. In this work, this ability was exploited to study the interactions between RSN communities at different spatial scales. DC measures the dependencies between two random variables $X$ and $Y$ with finite moments in arbitrary dimension [21]. For defining DC, let $(X, Y)=\left\{\left(X_{k}, Y_{k}\right) \mid k=1,2, \ldots, n\right\}$ an observed random sample of the joint distribution of random vectors $X$ in $\mathbb{R}^{p}$ and $Y$ in $\mathbb{R}^{q}$. Using these samples a transformed distance matrix $A$ can be defined as follows:

$$
a_{k l}=\left\|X_{k}-X_{l}\right\|, \quad \bar{a}_{k .}=\frac{1}{n} \sum_{l=1}^{n} a_{k l}, \quad \bar{a}_{\cdot l}=\frac{1}{n} \sum_{k=1}^{n} a_{k l}, \quad \bar{a}_{. .}=\frac{1}{n^{2}} \sum_{k, l=1}^{n} a_{k l}
$$

$A_{k l}=a_{k l}-\bar{a}_{k .}-\bar{a}_{. l}+\bar{a}_{. .}$, where $k, l=1,2, \ldots, n$. Similarly, $B$ is defined to characterize distances between samples for $Y$. An empirical distance between $X$ and $Y$ can be defined by

$$
V_{n}^{2}(X, Y)=\frac{1}{n^{2}} \sum_{k, l=1}^{n} A_{k l} B_{k l} \rightarrow R_{n}(X, Y)=\begin{gathered}
\frac{V_{n}^{2}(X, Y)}{\sqrt{V_{n}^{2}(X) V_{n}^{2}(Y)}} V_{n}^{2}(X) V_{n}^{2}(Y)>0 \\
0 \quad V_{n}^{2}(X) V_{n}^{2}(Y)=0
\end{gathered}
$$


where $V_{n}^{2}(X)=V_{n}^{2}(X, X)$. Note that $A$ and $B$ can be computed independently of $p$ and $q$, and both contain information about the distance between sample elements in $X$ and $Y . V_{n}^{2}(X, Y)$ is a measure of the distance between the probability distribution of the joint distribution and the product of the marginal distributions, i.e., $V_{n}^{2}(X, Y)$ quantifies $\left\|f_{X, Y}-f_{X} f_{Y}\right\|$, with $f_{X}$ and $f_{Y}$ the characteristic function of $X$ and $Y$, respectively, and $f_{X, Y}$ the joint characteristic function [21]. The DC corresponds to a normalized version of $V_{n}^{2}(X, Y)$, which takes values between 0 and 1 , with zero corresponding to statistical independence between $X$ and $Y$, and 1 total dependency.

To quantify the level of interactions among communities, previously the DC measure was obtained. For this, let $r=10$, the number of RSNs after applying the RSN identification process (see Section 2.2). Each community can be represented by the time-courses of their RSNs, i.e., $c_{i}=\left\{t_{1}, t_{2}, \ldots, t_{k_{i}}\right\}$ with $k_{i}$ the number of RSN that conform the ith-community and $C=\left\{c_{1}, c_{2}, \ldots c_{p}\right\}, p \leq r$ the set of emerging communities on fMRI signal for a particular subject, as shown in Figure 2A. The time-courses in the set $c_{i}$ can be used to build a multivariate random variable $Q_{i}$ by concatenating the set of RSN time courses in arbitrary order, with $Q_{i} \in \mathbb{R}^{k_{i} \times M}$, where $\mathrm{M}$ is the size of the time course. The interaction between pairs of communities was computed by using distance correlation. A total of $p ! /(2 !(p-2) !)$ possible pairs were assessed. The interaction between pairs of communities was defined as the distance correlation between the community $c_{i}$ and the community $c_{j}$, i.e., $R\left(Q_{i}, Q_{j}\right)$, see Figure $2 \mathrm{~B}$. This multivariate FNC approach was calculated at individual level using the communities previously found. A total of 15 interactions were studied.

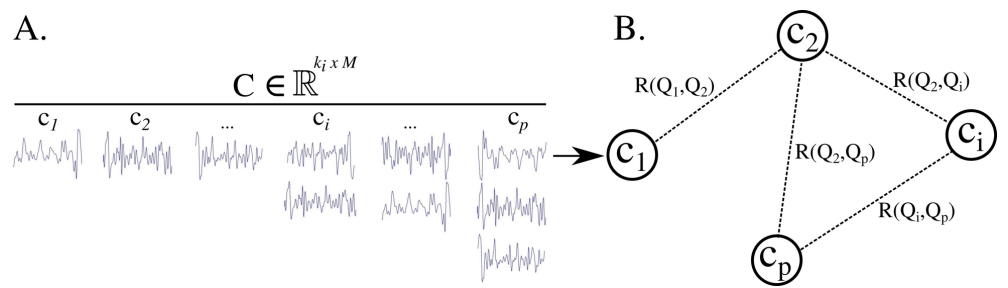

Fig. 2. Graph construction. Each community $c_{i}$ is composed of $k_{i}$ time-courses and they represent the nodes in the graph representation. The edges in the graph of communities, are the distance correlation measure $R$ between each possible relationship among communities.

\subsection{Group Analysis}

To quantify the capacity of the proposed method to characterize patients with DOC a group analysis was performed. In particular, the discrimination capacity between different diagnostic categories was evaluated in two settings. Firstly, in the characterization of differences between MCS vs VS/UWS subjects. Secondly, in the discrimination of healthy subjects and patients with DOC (MCS 
and VS/UWS). A Student's t-test was used to assess significant values of interaction at the group level $(p<0.05)$. Differences among healthy, VS/UWS and MCS subjects, were assessed by using a two sample t-test $(\mathrm{p}<0.0033)$, these computations were Bonferroni corrected to account multiple comparisons [7,22].

\section{Results}

Figure 3 shows the average of the multivariate FNC among communities for the three studied groups: healthy (left), MCS (middle) and VS/UWS (right) subjects. As observed, there is no a reconfiguration of the connectivity through the groups and the level of strength for some interactions changed in DOC conditions in contrast to healthy subjects, for instance, there is a reduction of the average functional connectivity between Visual System and Salience-Sensorimotor communities in VS/UWS patients in contrast to healthy subjects. In addition, there is an increase of the average functional connectivity between the auditory and the executive control communities in MCS patients in contrast to healthy subjects.

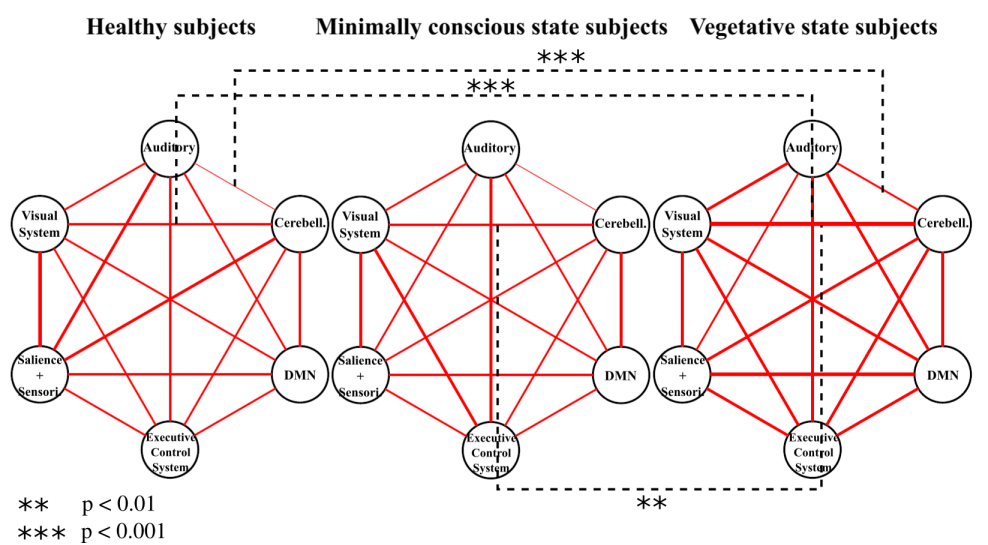

Fig. 3. Multivariate functional connectivity at group level. The line thickness represents the connectivity strength between two different communities.

Significant differences between VS/UWS and MCS patients were found for the relation between the visual system and cerebellum (relation marked with ${ }^{* *}$ in Figure 3). In addition, significant differences were found among healthy subjects and VS/UWS patients for the visual system - cerebellum and cerebellum - auditory connections (relation marked with $* * *$ in figure 3 ). These results suggest that an increase of FNC among different communities maybe linked to loss of consciousness, this is consistent with our previous results [7,22].

\section{Conclusions}

In this work, we proposed a novel strategy to study of brain dynamic in resting state by using a multivariate FNC approach. For this, a community or hyper- 
edge detection process was used to determinate the RSNs with similar behavior. Recently, similar approaches has been proposed $[13,14]$, however, have not been applied in the study of disorders of consciousness. On the other hand, in this work distance correlation measure was used to quantify the amount of interaction between different communities or hyperedge, probably into different spatial scale. Recently, analogous approach was proposed to redefine the functional network connectivity, using distance correlation to estimate the level of interaction based in all the signals from two regions of interest without the need to average over voxels first [23]. Finally, the proposed strategy was evaluated in patients with DOC. The results suggest that the new strategy improves the capacity of characterization of these patients. In particular, the interaction among visual system and cerebellum can be a potential biomarker for these conditions.

\section{Acknowledgment}

This work was supported by the James McDonnell Foundation, the European Space Agency, Mind Science Foundation, the French Speaking Community Concerted Research Action, the Belgian inter university attraction pole, the Public Utility Foundation "Université Européenne du Travail", "Fondazione Europea di Ricerca Biomedica" , the University Hospital of Liége and agreement Gobernación del Magdalena - COLCIENCIAS (Instituto Colombiano para el Desarrollo de la Ciencia y la Tecnologa).

\section{References}

1. Laureys, S. et. al., BMC Medicine, vol. 8, no. 1, pp. 1-4, 2010.

2. Giacino, J. et. al., Nature Reviews Neurology, vol. 10, p. 99 114, 2014.

3. Damoiseaux J. et. al., Proc. of the Nat. Academy of Sciences, vol. 103, no. 37, 2006.

4. Liu, X. et. al., Neuropsychiatr Dis Treat, vol. 11, pp. 2573-2581, 2015.

5. He, H. et. al., J Affect Disord, vol. 190, pp. 483-493, Jan 2016.

6. Dae-Jin K. et. al, NeuroImage, vol. 124, Part A, pp. 550 - 556, 2016

7. Rudas, J. and Guaje, J. et. al., Conf Proc IEEE Eng Med Biol Soc, 2014.

8. Rudas, J. et. al., Proc. SPIE, vol. 9681, pp. 96810 U-96 810U-9, 2015.

9. Hannawi, Y. et. al., Neurology, vol. 84, no. 12, pp. $1272-1280,2015$.

10. Kolchinsky, A. et. al., Front Neuroinform, vol. 8, p. 66, 2014.

11. Fujita, A. et. al., ArXiv e-prints, Dec. 2015.

12. S. Fortunato, CoRR, vol. abs/0906.0612, 2009. [Online]. Available: http://arxiv.org/abs/0906.0612

13. E. N. e. a. Davison, "," PLoS Comput. Biol., vol. 11, no. 1, p. e1004029, 2015.

14. B. e. a. Jie, "," Med Image Anal, vol. 32, pp. 84-100, 2016.

15. Schnakers, C. et. al., Brain Injury, vol. 20, no. 10, pp. 786-92, 2008.

16. Demertzi, A. and Gómez, F. et. al., Cortex, vol. 52, pp. 35-46, Mar 2014.

17. Cecile, B. et. al., Journal of Statistical Software, vol. 44, no. 9, 2011.

18. M. McKeown, Human Brain Mapping, vol. 6, pp. 160-188, 1998.

19. Kapur, J. et. al., C. Vis. Grap. and Image Proc., vol. 29, no. 3, pp. 273-285, 1985.

20. N. Otsu, IEEE Trans. on Sys., Man, and Cybern., vol. 9, no. 1, pp. 62-66, 1979.

21. Dae-Jin, K. et. al., The Annals of Statistics, vol. 35, no. 6, pp. 2769-2794, 122007.

22. Rudas, J. et. al., Proc. SPIE, vol. 9287, pp. $92870 \mathrm{P}-92870 \mathrm{P}-6,2015$.

23. L. G. et. al., NeuroImage, vol. 135, pp. 16-31, 2016. 